\title{
QT dispersion in sinus beats and ventricular extrasystoles in normal hearts
}

\author{
Christopher P Day, Janet M McComb, Ronald W F Campbell
}

\begin{abstract}
Objective-Recent studies have suggested that QT interlead variability (dispersion) on the surface electrocardiogram may have potential as a measure of recovery time dispersion. To test this hypothesis further QT dispersion occurring in sinus beats was compared with that in ventricular extrasystoles.
\end{abstract}

Design-Simultaneous electrocardiograms were recorded at $50 \mathrm{~mm} / \mathrm{s}$ during sinus rhythm in a drug free state while ventricular extrastimuli were introduced by programmed right ventricular stimulation at different coupling intervals. QT dispersion, defined as the difference between the maximum and minimum QT, was calculated separately for the extrasystoles and preceding and following sinus complexes. To correct for the influence of the number of measurable leads on QT dispersion, an "adjusted" QT dispersion calculated as QT dispersion/square root of the number of measurable leads, was used to compare sinus complexes and extrasystoles.

Patients-Nine patients were studied who were undergoing electrophysiological study for investigation of palpitation and were found to have electrically normal ventricles.

Results-At all coupling intervals tested "adjusted" QT dispersion was significantly greater in the ventricular extrasystoles than in either the preceding or following sinus complexes. For the coupling interval $350 \mathrm{~ms}$, the $95 \%$ confidence intervals for the difference between means was 52 to 78 ms (preceding sinus complex) and 56 to $82 \mathrm{~ms}$ (following sinus complex) $(p<0.00001)$. There was no correlation between the coupling interval and the magnitude of the "adjusted" QT dispersion.

Conclusion-These results accord fully with expected differences in ventricular recovery time dispersion and offer further support for the hypothesis that $Q T$ dispersion reflects regional variation in ventricular recovery. If substantiated by invasive studies, these findings have wide implications for both the usefulness and the method of QT measurement.
As early as 1887 the QT interval of the surface electrocardiogram was recognised as a potential measure of electrical recovery in the ventricles. ${ }^{1}$ Since then, prolongation of the QT interval has been associated with a risk of arrhythmias, ${ }^{2}$ a poor prognosis after myocardial infarction, ${ }^{3}$ and drug toxicity ${ }^{4}$ and has been regarded as evidence that a cardiac drug has reached its target organ. ${ }^{5}$ The QT interval in these circumstances is a measure of the time from the earliest ventricular depolarisation to the latest repolarisation. But both these electrical processes are naturally temporally dispersed. A wave of excitation is followed by a wave of recovery. In an elegant review, Krikler identified the significance of the R-on- $T$ ventricular extrasystole which not uncommonly complicates acute myocardial infarction and which shows that even early in the genesis of the surface electrocardiogram $T$ wave some myocardial areas have regained excitability. ${ }^{6}$ Such dispersion of repolarisation is widely acknowledged as a likely substrate for serious ventricular arrhythmias but its existence is not exposed by a simple single measure of QT interval.

Work in our department has addressed whether interlead QT variability on the surface electrocardiogram is reflecting variation in myocardial recovery of excitability. Strong support for that hypothesis has come from analysis of patients with arrhythmogenic QT prolongation and antiarrhythmic QT prolongation ${ }^{7}$ and also from survivors of acute myocardial infarction treated with placebo and sotalol. ${ }^{8}$ We now report the effect of ventricular extrasystoles on interlead QT varability.

\section{Patients and methods}

METHODS

We studied nine patients who were undergoing electrophysiological investigation of palpitation. All were found to have electrically normal ventricles: none had evidence of preexcitation and none had inducible ventricular tachycardia. Continuous simultaneous 12 lead electrocardiograms were recorded at $50 \mathrm{~mm} / \mathrm{s}$ during sinus rhythm in a drug free state while ventricular extrasystoles were introduced by programmed right ventricular stimulation either $300 \mathrm{~ms}$ or $350 \mathrm{~ms}$ after the preceding $Q$ wave. A wider range of coupling 
"Adjusted" QT dispersion (mean $(S D)$ ) in from preceding $Q$ wave) "Adjusted" dispersion was calculated as the difference minimum $Q T$ divided by the square root of the number of measurable leads. ventricular extrasystoles (coupling interval $350 \mathrm{~ms}$ and preceding and following sinus complexes. between maximum and

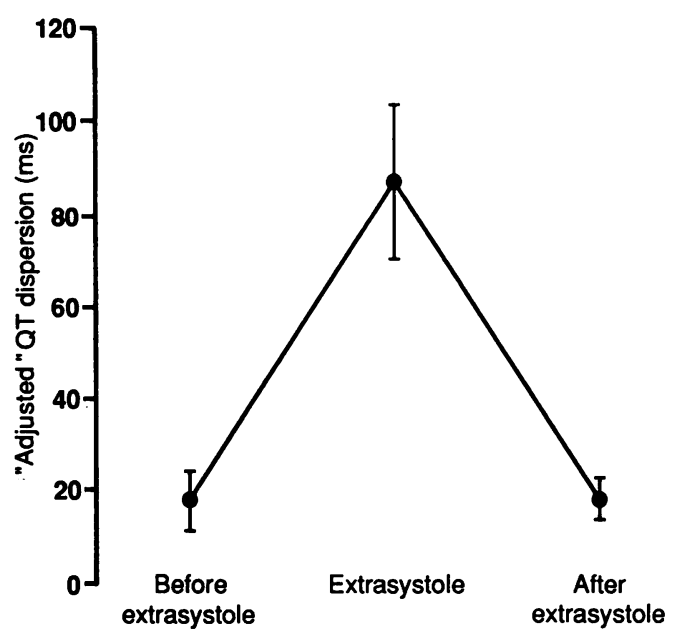

intervals from $280 \mathrm{~ms}$ to $400 \mathrm{~ms}$ was also tested in six of the patients. Ten sinus complexes separated the differently timed ventricular extrasystoles.

QT measurements were performed by one observer using a digitiser (CALCOMP 9000). All electrocardiograpic analysis was performed after the studies were completed. QT intervals were measured from the onset of the $Q R S$ to the end of the $T$ wave (defined as its return to the T-P baseline) in the last completed sinus complex preceding the ventricular extrasystole and the first sinus complex following the ventricular extrasystole. When $U$ waves were present, the QT was measured to the nadir of the curve between the $T$ and $U$ wave. For the ventricular extrasystoles the interval between the pacing spike and the end of the $T$ wave was measured. QT dispersion, defined as the difference between maximum and minimum QT, was calculated separately for the ventricular extrasystoles, the preceding sinus complexes and the following sinus complexes. QT measurements were performed only in those leads where the $T$ wave end could be reliably identified. In a previous study we showed that QT dispersion values are directly proportional to the square root of the number of measurable leads and derived an "adjusted" QT dispersion calculated as QT dispersion divided by the square root of the number of measurable leads to correct for this influence. ${ }^{8}$ Accordingly, for statistical analysis, this "adjusted" QT dispersion was calculated and used to compare the dispersion occurring in sinus complexes and ventricular extrasystoles.

Student's paired $t$ test was used to compared the mean "adjusted" QT dispersion occurring in sinus complexes and ventricular

Table 1 "Adjusted" $Q T$ dispersion (mean (SE)) in ventricular extrasystoles and preceding and following sinus complexes

\begin{tabular}{llll}
\hline & \multicolumn{2}{l}{ “Adjusted" $Q T$ dispersion (ms) } \\
\cline { 2 - 4 } $\begin{array}{l}\text { Coupling } \\
\text { interval }\end{array}$ & $\begin{array}{l}\text { Preceding sinus } \\
\text { complex }\end{array}$ & $\begin{array}{l}\text { Ventricular } \\
\text { extrasystole }\end{array}$ & $\begin{array}{l}\text { Following } \\
\text { sinus complex }\end{array}$ \\
\hline 300 & $22(2)$ & $80(4)$ & $23(6)$ \\
350 & $18(2)$ & $87(6)$ & $18(2)$ \\
\hline
\end{tabular}

"Adjusted" dispersion was calculated as the difference between maximum and minimum QT divided by the square root of the number of measurable leads.

The coupling interval is the interval between the ventricular extrasystole and preceding $Q$ wave. extrasystoles. Confidence intervals were calculated for the differences between means.

\section{Results}

Table 1 and the figure show the results of QT $\underline{\square}$ dispersion analysis of sinus complexes and ventricular extrasystoles for the coupling interval $350 \mathrm{~ms}$. The mean "adjusted" QT dispersion of the ventricular extrasystole was significantly greater than that of either the preceding or following sinus complex ( $\mathrm{p}<0.00001$ ) with $95 \%$ confidence intervals for the difference between means of 72 to $78 \mathrm{~ms}$ (preceding sinus complex) and 56 to $82 \mathrm{~ms}$ (following sinus complex). There was no difference in "adjusted" dispersion between preceding and following sinus complexes. Similar results were obtained for the coupling interval $300 \mathrm{~ms}$ (also shown in table 1).

Table 2 shows the results of "adjusted" QT dispersion occurring in ventricular extrasystoles at a range of different coupling intervals. There was no correlation between the "adjusted' QT dispersion and the coupling interval, although for each interval the dispersion of the ventricular extrasystole was at least three times greater than that of either the preceding or following sinus complex. In an attempt to correlate the magnitude of QT dispersion with the timing of the ventricular extrasystole in relation to the state of ventricular recovery we calculated the "prematurity index" expressed as the ratio of the coupling interval to the QT interval of the normal beat or R-R/QT ${ }_{\max }{ }^{9}$ for each electrocardiogram (data not shown). There was no correlation between this "prematurity index" and "adjusted" QT dispersion.

\section{Discussion}

These results are a third piece of evidence that interlead QT variability as measured on the 12 lead electrocardiogram is not merely a technical artefact but probably reflects dispersion of recovery of ventricular excitability. The adjus- 9 ted QT dispersion of ventricular estrasystoles far exceeded that of the preceding or succeeding sinus complexes. Ventricular extrasystoles $N$ most certainly involve dispersion and disrup- $N$ tion of activation, and some of the measured QT variation must be attributed to this aspect. The lack of a positive correlation between the ventricular extrasytole coupling interval and the measured dispersion suggests that activation disturbances were the predominant factor 7 contributing to the QT dispersion. This is supported by the fact that irrespective of short $\stackrel{\square}{\overparen{D}}$ coupling intervals producing what were $\mathbb{D}$ tantamount to $R$-on-T ventricular extrasystoles the configuration of induced extrasystoles 8 and stimulus to onset times were independent of the coupling interval. The increased dispersion of activation times will also lead to increased dispersion of action potential durations depending on electrical restitution, but if this were the major factor producing QT dispersion then dispersion would have been expected to vary with coupling interval. Our work in humans accords with animal studies in 
Table 2 "Adjusted" QT dispersion in ventricular extrasystoles at varying coupling intervals

\begin{tabular}{lllllll}
\hline \multicolumn{7}{l}{ “Adjusted” $Q T$ dispersion (ms) } \\
\cline { 2 - 7 } Coupling interval (ms) & Case 1 & Case 2 & Case 3 & Case 4 & Case 5 & Case 6 \\
\hline 280 & - & 118 & - & 78 & - & 72 \\
300 & 57 & 95 & - & 75 & 81 & 101 \\
320 & 72 & 120 & 72 & 95 & 101 & 57 \\
350 & 72 & 113 & 78 & 66 & 75 & 78 \\
360 & 78 & 124 & 107 & 72 & 95 & 121 \\
400 & 69 & 78 & 98 & 61 & 66 & 107 \\
\hline
\end{tabular}

See footnote to table 1 .
Thus $Q$ wave onset, like pacing spike onset, is virtually synchronous with the earliest onset of ventricular activation.

Late in the development of one of cardiology's classic tools we have found that the surface electrocardiogram may provide new information. All available evidence is that QT dispersion does reflect underlying regional variations in the recovery of ventricular excitability. If this view is more widely endorsed then a radical revision of QT measurements will be necessary. Classic studies from the past may not survive scrutiny. The only scientifically acceptable QT information that would be obtained from a 12 lead electrocardiogram would be either the maximum measured QT interval (implying a search for this on all 12 leads of the surface electrocardiogram) or dispersion of QT intervals. increased dispersion of action potential durations was responsible for the difference.

Our investigations showed that the QT dispersion of the first sinus complex after the ventricular extrasystole was almost identical to that of the sinus complex preceding the extrasystole. Thus despite the important electrical pertubations caused by the ventricular extrasystole, the electrophysiology of the ventricle returned to the pre-ventricular extrasystole conditions within a single cardiac cycle in these patients with normal hearts. This finding supports the concept of "T wave memory" whereby the characteristics of electrical recovery are retained except in the face of prolonged and consistent abnormalities of activation. ${ }^{11} 12$

The methods we used may have been flawed. Surface electrocardiographic QT measurement is subject to errors but previous work has shown that variation in interlead measurements far outweigh inaccuracies introduced by any other factor including interobserver variation. ${ }^{13}$ It was obviously impossible to blind the observer to whether the complex being measured was sinus or extrasystolic but the large differences in QT dispersion between the two types of complex seem unlikely to be explained by observer bias alone. There are potential problems in comparing the dispersion of sinus initiated QRS complexes with that of QRS complexes induced by a pacing spike. Unlike the pacing spike the onset of the $Q$ wave might be expected to vary between leads according to previously established varations in activation time ${ }^{14}{ }^{15} ; \mathrm{QT}$ dispersion would then reflect dispersion of action potential durations rather than dispersion of ventricular recovery time. Previous work, however, has shown interlead variation in $Q$ wave onset to be small compared with known regional differences in activation time, ${ }^{1316}$ which is considered to reflect the greater dependence of $Q$ wave onset on global rather than local cardiac events. ${ }^{16}$
We thank Dr Dennis M Krikler whose help, encouragement, and stimulation kindled our enthusiasm for this research. The work was supported by a grant from the British Heart Foundawork was supported by a grant from the B
tion and the British Digestive Foundation.

This work was presented, in part, at the British Cardiac Society in May 1990 in the final of the Young Investigators Award.

1 Waller $\mathrm{AD}$. A demonstration on man of electromotive changes accompanying the heart's beat. J Physics 1887;8:229.

2 Schwartz PJ, Periti M, Malliani A. The long Q-T syndrome. Am Heart J 1975;89:378-90.

3 Ahnve S, Helmers C, Lundman T, Rehnqvist N, Sjogren A. prognostic implications. Clin Cardiol 1980;1980;3:303-8.

4 Fraser AG, Ikram S. Torsade de pointes with prenylamine: do we still need the drug? Lancet 1986;ii:572

5 Edvardsson N, Hirsch I, Emanuelsson H, Ponten J, Olssen SB. Sotalol induced delayed ventricular repolarisation in man. Eur Heart $J$ 1980;1:335-43.

6 Krikler DM. A fresh look at cardiac arrhythmias. Pathogenesis and presentation. Lancet 1974;i:913-8.

7 Day CP, McComb JM, Campbell RWF. QT dispersion: an indication of arrhythmia risk in patients with long QT intervals. Br Heart $J$ 1990;63:342-4.

8 Day CP, McComb JM, Matthews J, Campbell RWF. Reduction in QT dispersion by sotalol following myocardial infarction. Eur Heart J 1991;12:423-7.

9 Lown B, Klein MD, Hershberg PI. Coronary and precoronary care. Am J Med 1969;46:705-24.

10 Kuo CS, Amlie JP, Munakata K, Reddy CP, Surawicz B. Dispersion of monophasic action potential durations and activation times during atrial pacing, ventricular pacing, activation times during atrial pacing, ventricular pacing, and ventricular premature stimulation
tricles. Cardiovasc Res 1983;17:152-61.

11 Franz MR, Bargheer K, Rafflenbuel W, Haverich A, Lichtlen PR. Monophasic action potential mapping in human subjects with normal electrocardograms: direct evidence for the genesis of the T wave. Circulation 1987;75:379-86.

12 Costard-Jöckle A, Franz MR. Dispersion of repolarisation followingm ectopic ventricular pacing [abstract]. Eur Heart $J$ 1989;10:24.

13 Cowan JC, Yusoff K, Moore M, et al. Importance of lead selection in QT interval measurement. Am J Cardiol 1988;61:83-7.

14 Cowan JC, Hilton CJ, Griffiths CJ, et al. Sequence of epicardial repolarisation and configuration of the $T$ wave epicardial repolarisation and

15 Spach MS, Barr RC. Ventricular intramural and epicardial repolarisation in the dog. Circ Res 1975;37:243-57.

16 Mirvis DM. Spatal variation of QT intervals in normal persons and patients with acute myocardial infarction. $J$ Am Coll Cardiol 1985;5:625-31. QTc intervals in acute myocardial infarction: first year potential distributions during ventricular activation and 